\title{
Decreased swallowing function in the sarcopenic elderly without clinical dysphagia: a cross-sectional study
}

\author{
Yen-Chih Chen ${ }^{1}$, Pei-Yun Chen ${ }^{1}$, Yu-Chen Wang ${ }^{1}$, Tyng-Guey Wang ${ }^{1 *}$ and Der-Sheng Han²
}

\begin{abstract}
Background: Sarcopenia and dysphagia are prevalent health issues as the elderly population continues to grow. However, whether sarcopenia, defined by either reduced handgrip strength or gait speed, would lead to pathological effects on swallowing function is still a matter of debate. Studies focusing on subclinical changes in the swallowing function in the sarcopenic elderly are lacking. This study evaluates the swallowing function in the sarcopenic elderly without dysphagia.

Methods: A cross-sectional study was conducted including subjects recruited from the community. Ninety-four individuals aged 65 and older without dysphagia were divided into two groups: sarcopenia and nonsarcopenia. The swallowing assessment included tongue pressure measurement, hyoid displacement (HD), hyoid velocity (HV) measurement with submental ultrasonography, 100-ml water-swallowing test, and the 10-item Eating Assessment Tool (EAT-10).

Results: The average tongue pressure was $47.0 \pm 13.7$ and $48.6 \pm 11.5 \mathrm{kPa}$ in the sarcopenia and nonsarcopenia groups, respectively $(p=0.55)$, whereas the average $\mathrm{HD}$ during swallowing was $15.3 \pm 4.4$ and $13.0 \pm 4.2 \mathrm{~mm}$ in the sarcopenia and nonsarcopenia groups, respectively $(p<0.05)$. The median of HV during swallowing was $19.5(6.41-$ 45.86) and $15.9(3.7-39.7) \mathrm{mm} / \mathrm{s}$ in the sarcopenia and nonsarcopenia group $(p<0.05)$. The median of time needed for consuming $100 \mathrm{ml}$ water was $12.43(3.56-49.34)$ and 5.66 (2.07-19.13) seconds in the sarcopenia and nonsarcopenia groups, respectively $(p<0.05)$. The median of the EAT-10 score was $0(0-2)$ and $0(0-1)$ in the sarcopenia and nonsarcopenia groups, respectively $(p<0.05)$.

Conclusions: In elderly individuals, swallowing function was significantly impaired with sarcopenia before clinical symptoms become clear. However, tongue muscles exhibited resistance to sarcopenia. We observed compensative strategies in patients with sarcopenia, such as reduced swallowing speed and increased hyoid bone movement.
\end{abstract}

Keywords: Swallow, Sarcopenia, Hyoid, Tongue, Dysphagia

\footnotetext{
* Correspondence: tgw@ntu.edu.tw

'Department of Physical Medicine and Rehabilitation, National Taiwan University Hospital, No.1, Changde Street, Zhongzheng District, Taipei City 10048, Taiwan, Republic of China

Full list of author information is available at the end of the article
}

C C The Author(s). 2020 Open Access This article is licensed under a Creative Commons Attribution 4.0 International License, which permits use, sharing, adaptation, distribution and reproduction in any medium or format, as long as you give appropriate credit to the original author(s) and the source, provide a link to the Creative Commons licence, and indicate if changes were made. The images or other third party material in this article are included in the article's Creative Commons. licence, unless indicated otherwise in a credit line to the material. If material is not included in the article's Creative Commons licence and your intended use is not permitted by statutory regulation or exceeds the permitted use, you will need to obtain permission directly from the copyright holder. To view a copy of this licence, visit http://creativecommons.org/licenses/by/4.0/ The Creative Commons Public Domain Dedication waiver (http://creativecommons.org/publicdomain/zero/1.0/) applies to the data made available in this article, unless otherwise stated in a credit line to the data. 


\section{Background}

Skeletal muscle mass decreases with advancing age, resulting in decreased strength and functionality. In 1989, Irwin Rosenberg first coined the term "sarcopenia" to describe this phenomenon [1]. Numerous research groups exist worldwide with varying definitions and diagnostic criteria for sarcopenia; however, it is generally agreed upon that sarcopenia has to be defined using a combined approach of muscle mass and muscle quality $[2,3]$. According to the Asian Working Group for Sarcopenia (AWGS) diagnostic criteria, sarcopenia should be described as low muscle mass plus low muscle strength (reduced handgrip strength) or low physical performance (reduced gait speed) [3].

Although the swallowing muscles are striated, their embryological characteristics are histologically different from those of the skeletal muscles of the trunk and extremities [4]. A previous study reported that swallowing muscles receive continuous input from the brainstem respiratory center and their activities are synchronized with the rhythmic contractions of the diaphragm [5]. Thus, sarcopenia, defined by the reduced handgrip strength and gait speed [3], does not necessarily lead to pathological effects on swallowing function.

The prevalence of sarcopenic dysphagia is still undetermined [4]. Recently, a growing number of studies demonstrated that sarcopenia might reduce not only body strength but also the strength of swallowing muscles, leading to swallowing function deterioration [6-10]. Most studies included hospitalized and facility-dwelling patients [6-9]; therefore, further studies are warranted involving other settings, such as the community setting. To the best of our knowledge, no studies targeted the swallowing assessment of sarcopenic patients without dysphagia. Discovering the characteristics indicative of the decline in swallowing function is of the utmost importance for providing the appropriate treatment before dysphagia becomes worse.

\section{Methods}

We used tongue pressure measurement, submental ultrasonography, 100-ml water-swallowing test (WST), and the 10-item Eating Assessment Tool (EAT-10) to evaluate the swallowing function in elderly sarcopenic patients without dysphagia. A cross-sectional study was conducted including 94 community-dwelling older individuals (ages above 65). An open announcement was made in Wanhua District, Taipei City, Taiwan, calling for joining the study. The participants were living independently and fully cooperative and were able to eat orally at the time of referral. Exclusion criteria were as follows: People who scored three points or higher on the EAT-10 [11]; those with a history of a neurological disorder such as cerebrovascular diseases, Parkinson's disease (PD), motor neuron disease, multiple sclerosis (MS), myopathy, and head and neck cancers. According to the AWGS definition, participants were assigned to two groups: sarcopenia or nonsarcopenia (47 individuals each) [3]. We utilized this definition because AWGS collected the best available evidence in sarcopenia research from eastern Asian countries and it was in line with previous reports describing sarcopenia as low muscle mass plus low muscle strength and/or low physical performance. The cutoff values for muscle mass measurements were $7.0 \mathrm{~kg} / \mathrm{m}^{2}$ and $5.4 \mathrm{~kg} / \mathrm{m}^{2}$ for men and women using dual X-ray absorptiometry and $7.0 \mathrm{~kg} / \mathrm{m}^{2}$ and $5.7 \mathrm{~kg} / \mathrm{m}^{2}$ for men and women using bioimpedance analysis, respectively, whereas the cutoff values for handgrip strength were $<26 \mathrm{~kg}$ and $<18 \mathrm{~kg}$ for men and women, respectively. The cutoff value for usual gait speed was $<0.8 \mathrm{~m} / \mathrm{s}$ and the gait speed was casual (at one's own pace).

Tongue muscle pressure has frequently been used as a measure of swallowing muscle strength [9, 12-15]. Iowa Oral Performance Instrument (IOPI) is one of the most widely used measurement techniques available for assessing the maximum pressure created by contact between the tongue and palate, objectively measuring tongue strength and endurance in practice $[13,16]$, with good inter- and intrarater reliability $[15,17]$. It is a handheld portable device using an air-filled pliable plastic tongue bulb connected with a clear plastic tube, placed on the middle part of the tongue, to estimate the peak pressure exerted on the tongue bulb.

Hyoid movement is essential for the adequate opening of the upper esophageal sphincter (UES) and measured by submental ultrasonography $[18,19]$. The protocol was applied and described in detail in previous works $[18,20]$ as follows. Each participant swallowed $3 \mathrm{~mL}$ of water three times where the hyoid bone movement was recorded during swallowing. Then, the best-recorded images of the three attempts were analyzed frame by frame. Moreover, the time interval from the swallowrelated hyoid motion onset to the first moment of maximum displacement in the forward movement trajectory was measured. We calculated the speed of hyoid movement as the maximal hyoid bone displacement divided by the time interval.

A $100 \mathrm{ml}$ WST is a sensitive predictor for identifying high-risk patients for swallowing dysfunction. Its sensitivity and specificity were $85.5 \%$ and was $50 \%$, respectively [21]. For the WST, we asked participants to consume $100 \mathrm{ml}$ of water in the shortest amount of time. Participants who choked during the test were asked to stop drinking immediately even if they had not finished the water; the protocol was detailed in previous works [21]. Furthermore, we calculated the swallowing speed, as the amount of consumed water divided by the elapsed time. 
The EAT-10 is a self-administered, symptom-specific outcome assessment for swallowing dysfunction, demonstrating good consistency, reproducibility, and validity [11]. It is comprised of 10 questions using a five-point Likert scale (0: no problem to 4: severe problem). The total score is ranging from 0 to 40 , with the higher scores indicating severe dysphagic symptoms.

We set the effect size as medium, the power as 0.8 , and the margin of error as $5 \%$ for sample size determination. Additionally, to calculate the sample size, we used G*Power software 3.1 [22]. Therefore, the number of participants required was 94 to an effect size of 0.52 . Statistical tests were conducted using IBM SPSS software (SPSS Statistics 20.0; SPSS Inc., Chicago, IL, USA). To assess the normality of data, we employed the Shapiro-Wilk test. The data of sarcopenia and nonsarcopenia groups were compared using a two-sample $t$-test if the values were normally distributed, or the MannWhitney $U$ test, otherwise. Based on the results of the normality test, the two-sample $t$-test was employed to detect the differences between the groups regarding the maximal pressure generated by contact between the tongue and palate (MTP) and hyoid displacement (HD) during swallowing, whereas the Mann-Whitney $U$ test was used for the differences between groups in terms of hyoid velocity (HV) during swallowing, the EAT-10 score, and the swallowing time obtained in the $100 \mathrm{ml}$ WST. The level of significance was 0.05 .

\section{Results}

Table 1 list the participants' characteristics. In the 94 subjects, the mean age was $75.1 \pm 5.8$ years and 26 (27.7\%) were men. Forty-seven of the ninety-four participants were in the sarcopenia group. No significant difference in age or sex was observed between the two groups.

Table 2 summarizes the swallowing factors in the sarcopenia and nonsarcopenia groups. According to the results of the normality test, MTP and HD complied with normal distribution, represented by mean followed by the standard deviation. However, EAT-10, WST, and HV did not comply with the normal distribution, represented by median followed by range. The EAT-10 score was significantly lower in the nonsarcopenia group than that of the sarcopenia group. The swallowing time for $100 \mathrm{ml}$ WST was significantly longer and the HD and HV during swallowing were significantly greater in the sarcopenia group than those in the nonsarcopenia group. No significant difference was observed regarding maximal tongue pressure between the two groups.

\section{Discussion}

In this study, we assessed the swallowing function in elderly sarcopenic patients without dysphagia. The EAT-10 score was significantly greater in the sarcopenia group than that in the nonsarcopenia group; however, despite the statistical difference, none of the participants' scores reached the cut point of 3 , suggesting dysphagia. Therefore, the differences in EAT-10 scores between the two groups should be interpreted with caution.

The time needed to consume $100 \mathrm{ml}$ of water was significantly longer in the sarcopenia group than that in the nonsarcopenia group, suggesting the swallowing function is reduced in patients with sarcopenia. Buchholz et al. postulated that in dysphagic patients, the swallowed bolus size might be reduced as a compensation strategy, resulting in a slower swallowing speed [23]. Nathadwarawala et al. discovered that, in subjects with swallowing problems, objective swallowing speed was significantly diminished [24]. Swallowing speed less than $10 \mathrm{ml} / \mathrm{s}$ is considered to be a strong predictor of dysphagia [25]. Although our sarcopenic participants have not developed dysphagia, the delay in completing the swallowing test reflects the compensatory mechanism (decreased volume per swallow) they developed before clinical problems become apparent [23, 24]. To the best of our knowledge, this is the first study to $100 \mathrm{ml} \mathrm{WST}$ on elderly individuals with sarcopenia. Yoshitoshi et al. evaluated the swallowing function in bedridden older adults using various bolus sizes (2, 3, and $5 \mathrm{ml}$ of liquid), where the swallowing performance was significantly correlated with the mid-upper arm circumference, rather than the general frailty [26]. The overall reduction of lean body mass might involve not only the mid-upper arm circumference but also the swallowing muscles.

The sarcopenia group had greater HD when swallowing $3 \mathrm{~mL}$ of water than the nonsarcopenia group. According to our knowledge, this is the first study

Table 1 Characteristics of the study population

\begin{tabular}{lll}
\hline & $\begin{array}{l}\text { Sarcopenia group }(\boldsymbol{n}=\mathbf{4 7}) \\
\text { Mean } \pm \text { SD }\end{array}$ & $\begin{array}{l}\text { Nonsarcopenia group }(\boldsymbol{n}=\mathbf{4 7}) \\
\text { Mean } \pm \text { SD }\end{array}$ \\
\hline Gender & $13(27.7 \%)$ & $13(27.7 \%)$ \\
Male (\%) & $34(72.3 \%)$ & $34(72.3 \%)$ \\
Female (\%) & $75.2 \pm 6.3$ & $75.1 \pm 5.4$ \\
\hline
\end{tabular}

A two-sample $t$-test was used to identify the differences between groups

SD standard deviation 
Table 2 Sarcopenia group and nonsarcopenia group outcomes

\begin{tabular}{lll}
\hline & Sarcopenia group $(\boldsymbol{n}=\mathbf{4 7})$ & Nonsarcopenia group $(\boldsymbol{n}=\mathbf{4 7})$ \\
\hline MTP $(\mathrm{kPa})$ & $47.0 \pm 13.7$ & $48.6 \pm 11.5$ \\
$\mathrm{HD}(\mathrm{mm})$ & $15.3 \pm 4.4$ & $13.0 \pm 4.2^{*}$ \\
HV $(\mathrm{mm} / \mathrm{s})$ & $19.5(6.41-45.86)$ & $15.9(3.7-39.7)^{*}$ \\
EAT-10 & $0(0-2)$ & $0(0-1)^{*}$ \\
WST (second) & $12.43(3.56-49.34)$ & $5.66(2.07-19.13)^{*}$ \\
\hline
\end{tabular}

MTP maximal pressure generated by contact between the tongue and palate, $H D$ hyoid displacement during swallowing, $H V$ hyoid velocity during swallowing, EAT-10 the 10-item Eating Assessment Tool score, WST swallowing time obtained in the 100 -ml water-swallowing test, ${ }^{*} p<0.05$

A two-sample $t$-test was used to identify the differences between groups in MTP and HD, while the Mann-Whitney $U$ test was used for HV, EAT-10, and WST

investigating the relationship between hyoid bone movement and sarcopenia. There was only one case report about a patient with sarcopenic dysphagia, reporting that both the maximal amounts of HD and HV during swallowing were increased after rehabilitation [27]. Previous research revealed that in an older population with dysphagia, HD was greater than the normal level in small bolus ingestion; however, the magnitude of HD decreased to normal or was below normal levels in large bolus ingestion [28]. It was hypothesized that greater displacement indicated compensation for insufficient UES opening, which could no longer be maintained with larger boluses in dysphagic patients [28]. Therefore, our findings included the clinical application of restricted bolus volume in sarcopenic patients with or without dysphagia. Meanwhile, rehabilitative programs for strengthening the hyoid elevation muscles might be useful [27].

Moreover, the sarcopenia group had greater HV when swallowing $3 \mathrm{~mL}$ of water than that in the nonsarcopenia group, which involves HD and duration. Previous studies hypothesized that $\mathrm{HV}$ is more sensitive in detecting changes in swallowing kinematics than HD alone [29] since studies of age-related changes in HD obtained heterogeneous results [30-32]. Furthermore, the decreased HV may slow down the closure of the laryngeal vestibule and opening of UES, thus increasing the risk of aspiration [33]. Many studies have already incorporated HV to describe hyoid excursion kinematics during swallowing [29, 33]. For instance, Ueda et al. [34] reported decreased hyoid excursion velocity in dysphagic patients; alternatively, previous studies showed that older healthy subjects had greater HV during swallowing than their younger counterparts $[29,35]$, supporting the concept of adaptation and compensation in the healthy older individuals. Greater $\mathrm{HV}$ in our sarcopenic participants may indicate a compensatory response to subclinical changes in the swallowing mechanism. These interpretations are speculative and require future experimental evaluation; however, the results of this study suggest that HV may be a sensitive indicator when assessing the effects of sarcopenia on swallowing performance.

Our results revealed that there was no significant difference between the sarcopenia group and their healthy counterparts regarding tongue pressure. Studies have reported that tongue strengthening could enhance the maximum peak lingual pressures in both healthy adults and dysphagic patients; however, whether the gain in lingual pressures generalizes to the functional improvement in swallowing is still undetermined [36, 37]. A previous study done in Japan demonstrated that reduced tongue pressure is correlated with older individuals with sarcopenia [9]; however, $42.3 \%$ of their subjects suffered from dysphagia, and all their subjects were hospitalized at the time of enrollment. Besides, they only included older subjects who were at least 75 years old. This study only enrolled community-dwelling elderly individuals without dysphagia. Another explanation was based on histological evidence as follows. The swallowing muscles and somatic muscles stem from different embryological origins and the swallowing muscles were continuously activated by the brainstem respiratory center [5]. Many common sarcopenic muscle characteristics are rare in the lingual muscles of rats, such as type II muscle fiber atrophy, and change to slower myosin heavy chain isoforms and neuromuscular junction dysmorphology [38, 39]. In particular, the styloglossus muscle was unaffected by sarcopenia anatomically and molecularly [40]. Besides, it was reported that the elderly can still produce similar noneffortful and effortful swallow pressures compared to younger adults [14] and tongue functional reserve does not decline significantly with age [41]. These findings suggest that tongue muscles are not as susceptible to sarcopenia as appendicular muscles and lingual function does not necessarily deteriorate with age.

This study had several limitations. First, we recruited our participants in annual health exams occurring in a local community hospital. Younger elderly individuals were more likely to receive recruiting information and join our study, whereas older people were less likely to be involved. Second, regarding WST, we did not measure the number of sips. Third, we did not assess the muscle mass related to swallowing. Fourth, we could not stratify the patients with sarcopenia according to the severity of the disease. Fifth, this study was conducted in a single region and included only community-dwelling elderly individuals without dysphagia within that 
location. A follow-up study including an expanded target area and sarcopenic patients with dysphagia is warranted.

\section{Conclusions}

Swallowing function is significantly reduced in elderly individuals with sarcopenia, even before the clinical symptoms become clear. However, tongue muscles appear to be resistant to sarcopenia. We observed compensative strategies in our sarcopenic participants, such as reduced swallowing speed and increased hyoid bone movement.

\section{Abbreviations}

AWGS: Asian Working Group for Sarcopenia; EAT-10: 10-item Eating Assessment Tool; MS: Multiple sclerosis; PD: Parkinson's disease; UES: Upper esophageal sphincter; WST: $100 \mathrm{ml}$ water-swallowing test

\section{Acknowledgements}

The authors would like to thank Bernice Yen for help with English usage and Ming-Chieh Shih for statistics help.

\section{Authors' contributions \\ Y.C.C participated in the data analysis and drafting the manuscript. D.S.H participated in the data collection. P.Y.C and Y.C.W participated in the statistical analysis. T.G.W revised the manuscript critically for important intellectual content. The author(s) read and approved the final manuscript.}

\section{Funding}

This study was sponsored by the Taiwanese Society of Physical Medicine and Rehabilitation, and Ministry of Science and Technology (MOST). The sponsor had no role in study design, data collection and analysis, decision to publish, or preparation of the manuscript.

\section{Availability of data and materials}

The datasets used and/or analyzed during the current study are available from the corresponding author on reasonable request.

\section{Ethics approval and consent to participate}

This study was approved by the ethics committee of National Taiwan University Hospital. Written informed consent was obtained from each participant.

\section{Consent for publication}

Not applicable.

\section{Competing interests}

All authors declare that they have no competing interests.

\section{Author details}

'Department of Physical Medicine and Rehabilitation, National Taiwan University Hospital, No.1, Changde Street, Zhongzheng District, Taipei City 10048, Taiwan, Republic of China. ${ }^{2}$ Physical Medicine \& Rehabilitation, National Taiwan University Hospital, Bei-Hu Branch, No.87, Neijiang Street, Wanhua District, Taipei City, Taiwan.

Received: 15 March 2020 Accepted: 13 October 2020

Published online: 21 October 2020

\section{References}

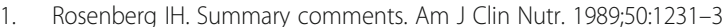

2. Cruz-Jentoft AJ, Baeyens JP, Bauer JM, Boirie Y, Cederholm T, Landi F, et al Sarcopenia: European consensus on definition and diagnosis: report of the European Working Group on sarcopenia in older people. Age Ageing. 2010; 39:412-23.

3. Chen LK, Liu LK, Woo J, Assantachai P, Auyeung TW, Bahyah KS, et al. Sarcopenia in Asia: consensus report of the Asian Working Group for sarcopenia. J Am Med Dir Assoc. 2014;15:95-101.
4. Fujishima I, Fujiu-Kurachi M, Arai H, Hyodo M, Kagaya $H$, Maeda K, et al. Sarcopenia and dysphagia: position paper by four professional organizations. Geriatr Gerontol Int. 2019;19:91-7.

5. Grelot L, Barillot JC, Bianchi AL. Pharyngeal motoneurones: respiratoryrelated activity and responses to laryngeal afferents in the decerebrate cat. Exp Brain Res. 1989;78:336-44.

6. Shiozu H, Higashijima M, Koga T. Association of sarcopenia with swallowing problems, related to nutrition and activities of daily living of elderly individuals. J Phys Ther Sci. 2015;27:393-6.

7. Maeda K, Akagi J. Sarcopenia is an independent risk factor of dysphagia in hospitalized older people. Geriatr Gerontol Int. 2016;16:515-21.

8. Wakabayashi H, Takahashi R, Murakami T. The prevalence and prognosis of sarcopenic dysphagia in patients who require dysphagia rehabilitation. J Nutr Health Aging. 2019;23:84-8.

9. Maeda K, Akagi J. Decreased tongue pressure is associated with sarcopenia and sarcopenic dysphagia in the elderly. Dysphagia. 2015;30:80-7.

10. Machida N, Tohara H, Hara K, Kumakura A, Wakasugi Y, Nakane A, et al. Effects of aging and sarcopenia on tongue pressure and jaw-opening force. Geriatr Gerontol Int. 2017:17:295-301.

11. Belafsky PC, Mouadeb DA, Rees CJ, Pryor JC, Postma GN, Allen J, et al. Validity and reliability of the eating assessment aool (EAT-10). Ann Otol Rhinol Laryngol. 2008;117:919-24

12. Sakai K, Nakayama E, Tohara H, Maeda T, Sugimoto M, Takehisa T, et al. Tongue strength is associated with grip strength and nutritional status in older adult inpatients of a rehabilitation hospital. Dysphagia. 2017;32:241-9.

13. Steele CM, Bailey GL, Molfenter SM, Yeates EM. Rationale for strength and skill goals in tongue resistance training: a review. Perspect Swallowing Swallowing Disord. 2009;18:49-54.

14. Yeates E, Steele C, Pelletier C. Tongue pressure and submental surface electromyography measures during noneffortful and effortful saliva swallows in healthy women. Am J Speech Lang Pathol. 2010;19:274-81.

15. Youmans SR, Stierwalt JA. Measures of tongue function related to normal swallowing. Dysphagia. 2006;21:102-11.

16. Adams V, Mathisen B, Baines S, Lazarus C, Callister R. A systematic review and meta-analysis of measurements of tongue and hand strength and endurance using the lowa oral performance instrument (IOPI). Dysphagia. 2013;28:350-69.

17. Youmans SR, Youmans GL, Stierwalt JA. Differences in tongue strength across age and gender: is there a diminished strength reserve? Dysphagia. 2009:24:57-65.

18. Chen YC, Hsiao MY, Wang YC, Fu CP, Wang TG. Reliability of ultrasonography in evaluating hyoid bone movement. J Med Ultrasound. 2017;25:90-5.

19. Ekberg O. The normal movements of the hyoid bone during swallow. Investig Radiol. 1986:21:408-10.

20. Chen YC, Hsiao MY, Chen PY, Wang TG. Effects of age on the speed of hyoid bone movement during swallowing. Int J Gerontol. 2019:13:344-8.

21. Wu MC, Chang YC, Wang TG, Lin LC. Evaluating swallowing dysfunction using a 100-ml water swallowing test. Dysphagia. 2004;19:43-7.

22. Faul F, Erdfelder E, Lang AG, Buchner A. G*Power 3: a flexible statistical power analysis program for the social, behavioral, and biomedical sciences. Behav Res Methods. 2007;39:175-91.

23. Buchholz DW, Bosma JF, Donner MW. Adaptation, compensation, and decompensation of the pharyngeal swallow. Gastrointest Radiol. 1985; 10:235-9.

24. Nathadwarawala KM, McGroary A, Wiles CM. Swallowing in neurological outpatients: use of a timed test. Dysphagia. 1994;9:120-9.

25. Nathadwarawala KM, Nicklin J, Wiles CM. A timed test of swallowing capacity for neurological patients. J Neurol Neurosurg Psychiatry. 1992;55: $822-5$.

26. Kuroda $Y$, Kuroda R. Relationship between thinness and swallowing function in japanese older adults: implications for sarcopenic dysphagia. J Am Geriatr Soc. 2012;60:1785-6

27. Nakayama E, Tohara H, Sato M, Hino H, Sakai M, Nagashima $Y$, et al. Time course and recovery of the movements of hyoid bone and thyroid cartilage during swallowing in a patient with sarcopenic dysphagia: a case report. Am J Phys Med Rehabil. 2019;99:e64-7.

28. Kendall KA, Leonard RJ. Hyoid movement during swallowing in older patients with dysphagia. Arch Otolaryngol Neck Surg. 2001;127:1224-9.

29. Barikroo A, Carnaby G, Crary M. Effects of age and bolus volume on velocity of hyolaryngeal excursion in healthy adults. Dysphagia. 2015;30:558-64. 
30. Nishikubo K, Mise K, Ameya M, Hirose K, Kobayashi T, Hyodo M. Quantitative evaluation of age-related alteration of swallowing function: videofluoroscopic and manometric studies. Auris Nasus Larynx. 2015;42:134-8.

31. Kim Y, McCullough GH. Maximum hyoid displacement in normal swallowing. Dysphagia. 2008;23:274-9.

32. Kang BS, Oh BM, Kim IS, Chung SG, Kim SJ, Han TR. Influence of aging on movement of the hyoid bone and epiglottis during normal swallowing: a motion analysis. Gerontology. 2010;56:474-82.

33. Ueda N, Nohara K, Kotani Y, Tanaka N, Okuno K, Sakai T. Effects of the bolus volume on hyoid movements in normal individuals. J Oral Rehabil. 2013;40:491-9.

34. Ueda N, Nohara K, Tanaka N, Kaneko N, Sakai T. A comparison of the maximum hyoid velocity in healthy adults and dysphagic patients. Dysphagia. 2013;28:646.

35. Chi-Fishman G, Sonies BC. Effects of systematic bolus viscosity and volume changes on hyoid movement kinematics. Dysphagia. 2002;17:278-87.

36. McKenna VS, Zhang B, Haines MB, Kelchner LN. A systematic review of isometric lingual strength-training programs in adults with and without dysphagia. Am J Speech Lang Pathol. 2017;26:524-39.

37. Smaoui $S$, Langridge A, Steele $C$. The effect of lingual resistance training interventions on adult swallow function: a systematic review. Dysphagia. 2019:14:1-7.

38. Connor NP, Russell JA, Wang H, Jackson MA, Mann L, Kluender K. Effect of tongue exercise on protrusive force and muscle fiber area in aging rats. J Speech Lang Hear Res. 2009;52:732-44.

39. Hodges SH, Anderson AL, Connor NP. Remodeling of neuromuscular junctions in aged rat genioglossus muscle. Ann Otol Rhinol Laryngol. 2004; 113:175-9.

40. Sokoloff AJ, Douglas M, Rahnert JA, Burkholder T, Easley KA, Luo Q. Absence of morphological and molecular correlates of sarcopenia in the macaque tongue muscle styloglossus. Exp Gerontol. 2016;84:40-8.

41. Steele CM. Optimal approaches for measuring tongue-pressure functional reserve. J Aging Res. 2013;2013:542909.

\section{Publisher's Note}

Springer Nature remains neutral with regard to jurisdictional claims in published maps and institutional affiliations.

Ready to submit your research? Choose BMC and benefit from:

- fast, convenient online submission

- thorough peer review by experienced researchers in your field

- rapid publication on acceptance

- support for research data, including large and complex data types

- gold Open Access which fosters wider collaboration and increased citations

- maximum visibility for your research: over $100 \mathrm{M}$ website views per year

At $\mathrm{BMC}$, research is always in progress.

Learn more biomedcentral.com/submissions 